\title{
To a question on possibilities of high-resolution NMR spectroscopy in the Earth magnetic field
}

Pavel Kupriyanov ( $\sim$ p.kupriyanov@spbu.ru )

Saint Petersburg State University: Sankt-peterburgskij gosudarstvennyj universitet

Vladimir Kirilenko

"Vorobyovy Gory" Technical Education Centre, Moscow

Anna Chizhik

Saint-Petersburg State University: Sankt-peterburgskij gosudarstvennyj universitet

Vladimir Chizhik

Saint-Petersburg State University: Sankt-peterburgskij gosudarstvennyj universitet

\section{Research Article}

Keywords: low signal-to-noise ratio, magnetic fields, NMR, spectroscopy

Posted Date: June 15th, 2021

DOI: https://doi.org/10.21203/rs.3.rs-560232/v1

License: (c) (i) This work is licensed under a Creative Commons Attribution 4.0 International License. Read Full License 


\section{Abstract}

The low signal-to-noise ratio is a characteristic feature of NMR experiments in the Earth magnetic field. The main problem of such studies is the ineffectiveness of signal accumulation due to fluctuations of the Earth magnetic field because of magnetic interferences from laboratory equipment and other magnetic field sources. Taking into account the fact that generally in the Earth magnetic field proton-containing liquids have spectra with a central strong line, a relatively simple method has been found to neutralize the Larmor frequency fluctuations. The NMR signal processing algorithm is described. The home-built NMRequipment allows us to clearly register the splitting in proton spectra, for example, due to the $J$-interaction with such nuclei as ${ }^{29} \mathrm{Si}$ or ${ }^{13} \mathrm{C}$ at their natural abundance (4.7 and $1.1 \%$, respectively).

\section{Introduction}

The basics of registration and features of nuclear magnetic resonance in the Earth magnetic field are described in detail, for example, in the monograph [1]. In 1957 D. Elliot and R. Schumacher obtained the ${ }^{1} \mathrm{H}$ signal from fluorobenzene in the Earth magnetic field [2], using the method of M. Packard and R. Varian $[3,4]$, and observed "slow beats" in the decay of the signal. They estimated the frequency of these beats and concluded that the $\mathrm{J}$-coupling constant for the ${ }^{19} \mathrm{~F}$ nuclei and protons is $5.8 \mathrm{~Hz}$. This was one of the first attempts to obtain the information about NMR spectrum by pulse method. However, already in the next 1958 in Russia at the Faculty of Physics of the Leningrad (now St. Petersburg) State University, F. Skripov and co-workers managed to obtain the proton spectrum of tributyl phosphate in the Earth magnetic field via the Fourier transform of a free induction decay (FID) [5]. However, they calculated the spectrum manually using electromechanical counting machines for many days and this circumstance explains the fact that their work did not attract the attention of contemporaries (see details in [6]).

With the advent of the possibility of creating magnetic systems with high field homogeneity, the interest of researchers to low-field NMR decreased significantly. The lack of chemical shifts in NMR spectra in weak fields such as the Earth's one and the low signal-to-noise ratio were also superimposed on this. Nevertheless, the works of P. Callaghan [7, 8] and S. Appelt [9-11] stand out, showing that despite of the absence of chemical shifts, NMR spectra can be informative, as they carry information about $J$-coupling constants. To obtain them, the high homogeneity of a static magnetic field is required, which is possessed only by very expensive and sophisticated devices, but in the Earth field narrow spectral lines can be obtained using home-built equipment. However, P. Callaghan limited himself to the study of fluorine-containing liquids with a high natural abundance of ${ }^{19} \mathrm{~F}$ magnetic nuclei $(100 \%)$, the interaction with which split proton spectra. S. Appelt used a strong Halbach magnet for pre-polarization of nuclei and manually transferred the sample in the NMR sensor, losing 1-2 seconds, but obtaining proton spectra contained lines due to $\mathrm{J}$-coupling with ${ }^{29} \mathrm{Si}(4.7 \%)$ and ${ }^{13} \mathrm{C}(1.1 \%)$ nuclei of natural abundance.

The main problem of such a study is the ineffectiveness of signal accumulation due to fluctuations of the Earth magnetic field because of magnetic interferences from laboratory equipment and other magnetic field sources. The original solution to the problem was proposed by J. Stepišnik and co-workers 
[12], who suggested using a second sensor to monitor changes of the resonance frequency caused by fluctuations in the Earth field. They used a signal from the second sensor with a water sample for the quadrature detection of a signal from the test sample. The authors [12] used their approach for MRI in the Earth field and the duration of the water signal was sufficient for the detection of short signals in the gradient field. We improved this method for the high-resolution NMR spectroscopy in the Earth field [13] by suggesting that after simultaneous recording of signals from both sensors one can determine the resonance frequency using the signal from the reference sensor and then forming a "infinite" reference signal at this frequency it is possible to detect the signal from the studied substance. However, taking into account the fact that generally in the Earth magnetic field proton-containing liquids have spectra with a central strong line, a simpler method has been found to neutralize the Larmor frequency fluctuations. The frequency of this line can be determined and then the total NMR signal can be processed using the reference signal with obtained frequency. After the fast Fourier transform (FFT) the individual spectra can be accumulated to increase the signal-to-noise ratio. The results of the application of this simple technique, combined with conventional NMR signal processing, are presented in the article.

\section{Notations}

The following notations will be applied within the scope of the article:

\begin{tabular}{ll}
$X_{k}$ & -th element of an any array \\
\hline$F$ & An array of discrete frequencies of a spectrum \\
\hline$A(\operatorname{Re} A, \operatorname{Im} A)$ & An array of discrete complex amplitudes of a spectrum \\
\hline$V$ & An array of discrete amplitudes of a signal
\end{tabular}

\section{Signal processing}

First, it is necessary to perform the FFT of a NMR signal (FID), and then the region of the spectrum with a strong line is selected and its frequency is calculated. However, the "straightforward" determination of the dominant frequency is not accurate enough. To achieve the necessary precision, it is necessary to perform the inverse FFT (IFFT) of a narrow segment of the spectrum with the strong line, thereby performing the narrow-band filtering of the signal, and then calculate the frequency. After that, the quadrature detection can be used for the original signal with the reference signal at the calculated frequency and it is possible to perform the FFT again. Thus, the NMR frequency of the strongest line in a spectrum shifts to zero. All subsequent signals processed in this way can be accumulated in the traditional manner (see Fig. 1). A demonstration of the prospects for registering spectra using the proposed algorithm for neutralizing fluctuations in the Earth magnetic field is shown in Figure 2.

Note that the spectrum does not have to contain only one strong line. If there are several strong lines in a spectrum, it is also possible to use one of the strong spectral lines to neutralize the frequency fluctuations. 
The NMR signal in the Earth magnetic field is recorded not immediately after the end of polarization (in the case of the Packard-Varian method [2, 3]) or after the 90-degree RF-pulse, but after the so-called "dead time" (time of receiver insensitivity). The problem is especially important in the case of the Earth field because of a very low resonance frequency (about $2 \mathrm{kHz}$ ) and, as a result, a long transient process in the receiving path ("dead time" is about 30-50 ms). In this case, the loss of the initial phase of a signal (FID) leads to the distortion of a spectrum. This effect varies for different frequencies, so it is impossible to align lines simultaneously for corrected spectrum element $C_{k}$ using the traditional method:

$$
C_{k}=\operatorname{Re} A_{k} \cos \phi+\operatorname{Im} A_{k} \sin \phi,
$$

where $\varphi$ is the handpicked variable. It is necessary to take into account the phase shift, which occurs due to the introduction of "dead time" in Eq. (1):

$$
C_{k}=\operatorname{Re} A_{k} \cos \left(2 \pi F_{k} t_{d}\right)+\operatorname{Im} A_{k} \sin \left(2 \pi F_{k} t_{d}\right),
$$

where $t_{d}$ is the "dead time". But in this case, oscillations are observed in the spectrum even if the apodization of a signal is done (as after adding zeros to a signal in the absence of apodization, see the details in Ref. [14]). The fact is that introducing the variable $t_{d}$ in Eq. (2) is equivalent to adding zeros at the beginning of a signal, emulating the segment of the "dead time" $t_{d}$. Fig. 3 (left) shows the "raw" spectrum of 2,2,2 trifluoroetanol, and Fig. 3 (right) shows the spectrum after applying Eq. (2). In other words, the oscillations appeared in the spectrum because entering $t_{d}$ into formula (2), we had actually shifted a signal along the time axis to the right, and zeros appeared in the time interval from zero to the beginning of a signal. This means that there is now a sharp front at the beginning of a signal, which causes the oscillations in the spectrum.

To increase the resolution in the spectrum, it is necessary to supplement the signal with zeros (see, for example, [1]). However, due to the presence of noise in the signal, a front appears at the boundary of the signal with the "zero part", causing oscillations in the spectrum (similar to Fig. 3, right). To eliminate this effect, the signal apodization procedure is used, which consists in multiplying the signal by a function that decreases to zero. Possible variants of these functions are described in Ref. [14].

\section{Equipment}

In all experiments, a home-built NMR spectrometer in the Earth magnetic field was used. A coil of special geometry with 1800 turns of copper wire with a diameter of $0.9 \mathrm{~mm}$ is used as a sensor (see Fig. 4, left). The internal diameter of the coil is $55 \mathrm{~mm}$, which allows the use of samples with a volume of about 130 $\mathrm{ml}$. The sensor is placed inside a shielding cube with aluminum walls measuring $450 \times 450 \mathrm{~mm}, 7 \mathrm{~mm}$ thick. The electronics consists of a preamplifier with a gain of about 1000, a tunable narrow-band biquadratic filter, and a switching unit allowing preliminary polarization by connecting a current source to the sensor coil. A current of 1.5 A was used for pre-polarization (pre-polarization field is about $20 \mathrm{mT}$ ). 
The bandwidth of the biquadratic filter is selected close to the bandwidth of the input circuit $(\approx 50 \mathrm{~Hz})$. A modified for low field NMR analyzer Spin Track from Resonance Systems Ltd (http://nmr-design.com) was used as a data collection system.

A frame for the sensor has the shape of an elongated parallelepiped. This shape reduces the size of the excitation coils and the field inhomogeneity compensation coils (see Fig. 4, right). The elongated rectangular shape of the coils is chosen because of cylindrical samples used. To calculate the optimal coil fields, the problem of four parallel current-carrying conductors was used.

For the compensation of local inhomogeneity in the first approximation the simple pair of "rectangular Maxwell coils" with an axis directed along the vector of the Earth magnetic field is used. The peculiarities of creating magnetic field gradients in weak fields are considered in the work [15]. An example of a signal in ordinary laboratory conditions is demonstrated in Fig. 5.

\section{Experiments}

The sensitivity of NMR devices in the Earth magnetic field does not allow one to clearly register the splitting in proton spectra due to the $\mathrm{J}$-interaction with such nuclei as ${ }^{29} \mathrm{Si}$ or ${ }^{13} \mathrm{C}$ at their natural abundance by the single scan method. The method described above for neutralizing fluctuations in the Earth magnetic field allowed us to make the necessary accumulations. In the case of the isotope ${ }^{29} \mathrm{Si}$ (natural abundance is $4.7 \%$ ) the 20 -fold signal accumulation is sufficient to reliably register of $J$-splittings in many silicon-containing liquids. For example, such similar liquids as hexamethyldisilazane and hexamethyldisiloxane differ slightly in J-coupling constant (see Fig. 6).

A relatively viscous grease polymethyl siloxane (PMS), when heated to $60^{\circ} \mathrm{C}$ (to decrease its viscosity), gives the spectrum in which the different $J$-splittings due to long-range interactions of protons and silicon nuclei appears (see Fig. 7).

The reliable registration of spectra with the lines due to the interaction of protons with ${ }^{13} \mathrm{C}$ nuclei with natural abundance (1.1\%) was our main goal. After processing batch of spectra with the described algorithm with the neutralization of frequency fluctuations, it was possible to obtain the desirable result. The spectra of acetone, acetic acid, and benzene are presented in Fig. 8. In all spectra, satellites are detected near the strongest line. The amplitude of the satellites correlates with the natural abundance of the ${ }^{13} \mathrm{C}$ nuclei. The Fig. 8 shows the $J$-constants that agree with the reference data. The splitting constants for the interaction of the neighboring pairs of nuclei ${ }^{1} \mathrm{H}-{ }^{13} \mathrm{C}$ are more than $100 \mathrm{~Hz}$ and the corresponding spectral lines lie outside the given frequency interval.

\section{Conclusion}


Currently, low field NMR spectroscopy is significantly inferior to high field spectroscopy in terms of its application for solving a wide range of analytical problems. However, despite the absence of chemical shifts, the NMR spectra in the Earth field have sufficient information content and frequency resolution to measure $J$-constants and identify liquids in some important cases. For example, it is advisable to consider the applicability of high-resolution NMR spectroscopy in the Earth magnetic field for the detection and determination of specific liquids (primarily illicit) during inspections, since this method allows obtaining NMR signals from large sealed samples (bottles, cans, cans, etc. etc.), including those with a thin metal diamagnetic shell $[17,18]$.

The low signal-to-noise ratio is a characteristic feature of NMR experiments in the Earth magnetic field. The main problem of such studies is the ineffectiveness of simple signal accumulation due to fluctuations of the Earth magnetic field because of magnetic interferences from laboratory equipment and other magnetic field sources. Taking into account the fact that generally in the Earth magnetic field the proton-containing liquids have spectra with a central strong line, a relatively simple method has been found to neutralize the Larmor frequency fluctuations. To achieve the necessary precision, it is necessary to perform the inverse FFT of a narrow segment of the spectrum with the strong line, thereby performing the narrow-band filtering of the signal, and then calculate the frequency. After that, the quadrature detection can be used for the original signal with the reference signal at the calculated frequency and it is possible to perform the FFT again. Thus, the NMR frequency of the strongest line in a spectrum shifts to zero. All subsequent signals processed in this way can be accumulated in the traditional manner (see Fig. 1 and 2). The use of the developed algorithm for neutralizing fluctuations of the Earth magnetic field made possible multiple accumulations of signals and reliable registration of satellites in spectra of protons due to their $\mathrm{J}$-interaction with such nuclei as ${ }^{29} \mathrm{Si}$ and ${ }^{13} \mathrm{C}$ at their natural abundance.

\section{Declarations}

Competing interests: The authors declare no competing interests.

\section{References}

1. V. Chizhik, Y. Chernyshev, A. Donets, V. Frolov, A. Komolkin, M. Shelyapina, Magnetic Resonance and Its Applications (Springer-Verlag., 2014), 782 pp. doi: 10.1007/978-3-319-05299-1

2. D. Elliott, R. Schumacher, The Journal of Chemical Physics, p. 1350, 26 (1957)

3. M. Packard, R. Varian, Bull. Amer. Phys. Soc. p. 941, (1953)

4. M. Packard, R. Varian, Phys. Rev. p. 941, 93 (1954)

5. Morozov, A. Mel'nikov, F. Skripov, Bulletin of the Academy of Sciences of the USSR, p. 1127, 22 (1958)

6. V. Chizhik, Bulletin du Groupement AMPERE, pp. 5-6 273 (2018)

7. J. Robinson, A. Coy, R. Dykstra, C. Eccles, M. Hunter, P. Callaghan, Journal of Magnetic Resonance, pp. 343-347, $182(2006)$ 
8. M. Halse, P. Callaghan, B. Feland, R. Wasylishen, Journal of Magnetic Resonance, pp. 88-94, 200 (2009)

9. S. Appelt, H. Kühn, F. Häsing, U. Sieling, B. Blümich, Nature physics, pp. 106-109, 2 (2006)

10. S. Appelt, F. Häsing, H. Kühn, B. Blümich, Phys. Rev. C 023420, 76 (2007)

11. S. Appelt, F. Häsing, H. Kühn, U. Sieling, B. Blümich, Chemical Physics Letters. p. 144, 440 (2007)

12. A. Mohorič, G. Planinšič, M. Kos, A. Duh, J. Stepišnik, Instrumentation science and technology, pp. 665-667, 6 (2004)

13. V. Chizhik, P. Kupriyanov, Eurasian patent №034623 (2020), available at: http://www.eapatis.com/Data/EATXT/eapo2020/PDF/034623.pdf

14. R. Ernst, G. Bodenhausen, A. Wokaun, Principles of Nuclear Magnetic Resonance in One and Two Dimensions (Brandeis University, 1990) 610 pp.

15. V. Chizhik, V. Frolov, P. Kupriyanov, K. Tyutyukin, App. Mag. Res. pp. 687-698, T. 48,7 (2017)

16. L. Chen, Z. Weng, L. Goh M. Garland, Journal of Magnetic Resonance, pp. 164-168, 158 (2002)

17. V. Chizhik, P. Kupriyanov, V. Mozzhukhin, Nanotechnology in the Security Systems, pp. 151-164, (2014)

18. E. Balcı, B. Rameev, H. Acar, G. Mozzhukhin, B. Aktaş, B. Çolak, P. Kupriyanov, A. levlev, Y. Chernyshev, V. Chizhik, App. Mag. Res. pp. 87-99, 47, (2016)

\section{Figures}




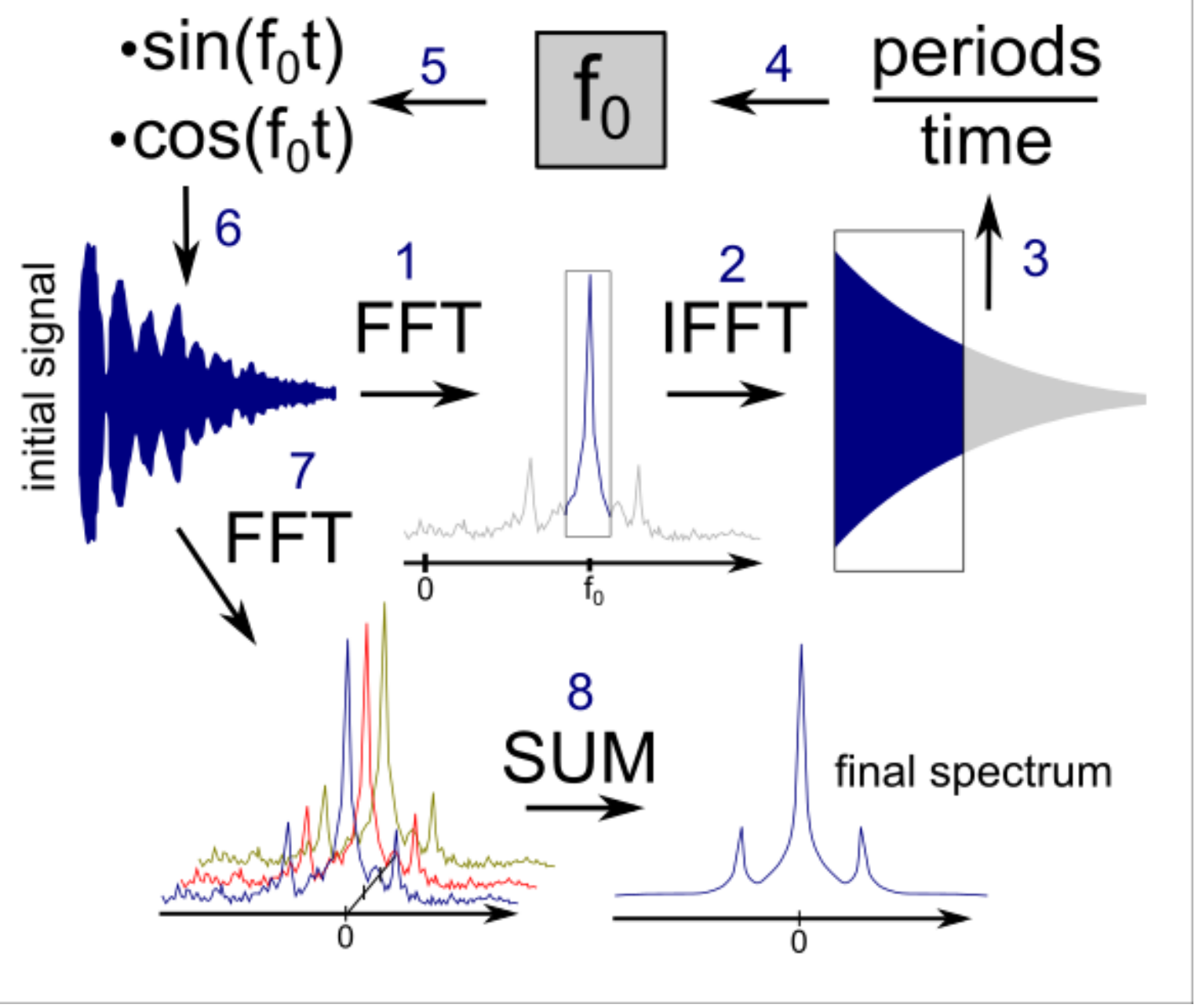

Figure 1

The algorithm of the neutralization of resonance frequency fluctuations for NMR in the Earth magnetic field. 


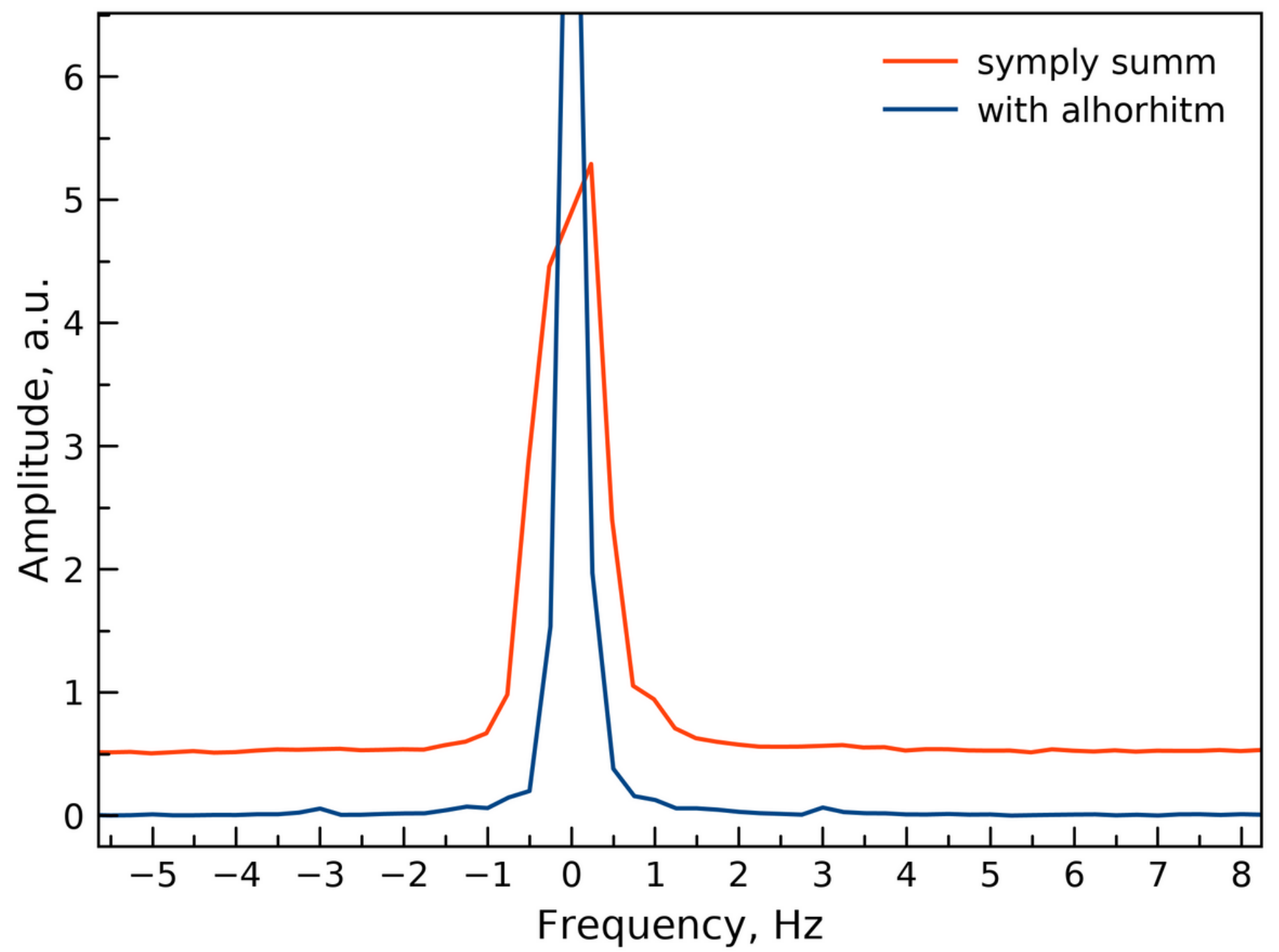

Figure 2

The NMR spectrum of acetone in the Earth magnetic field (averaging over 50 scans): the spectrum obtained with the neutralization of frequency fluctuations (blue) and the ordinary FFT of the sum of original signals (red). One can see the doublet (blue spectrum) at the $\pm 3.1 \mathrm{~Hz}$ due to J-coupling with $13 \mathrm{C}$ at the natural abundance (1.1\%). 

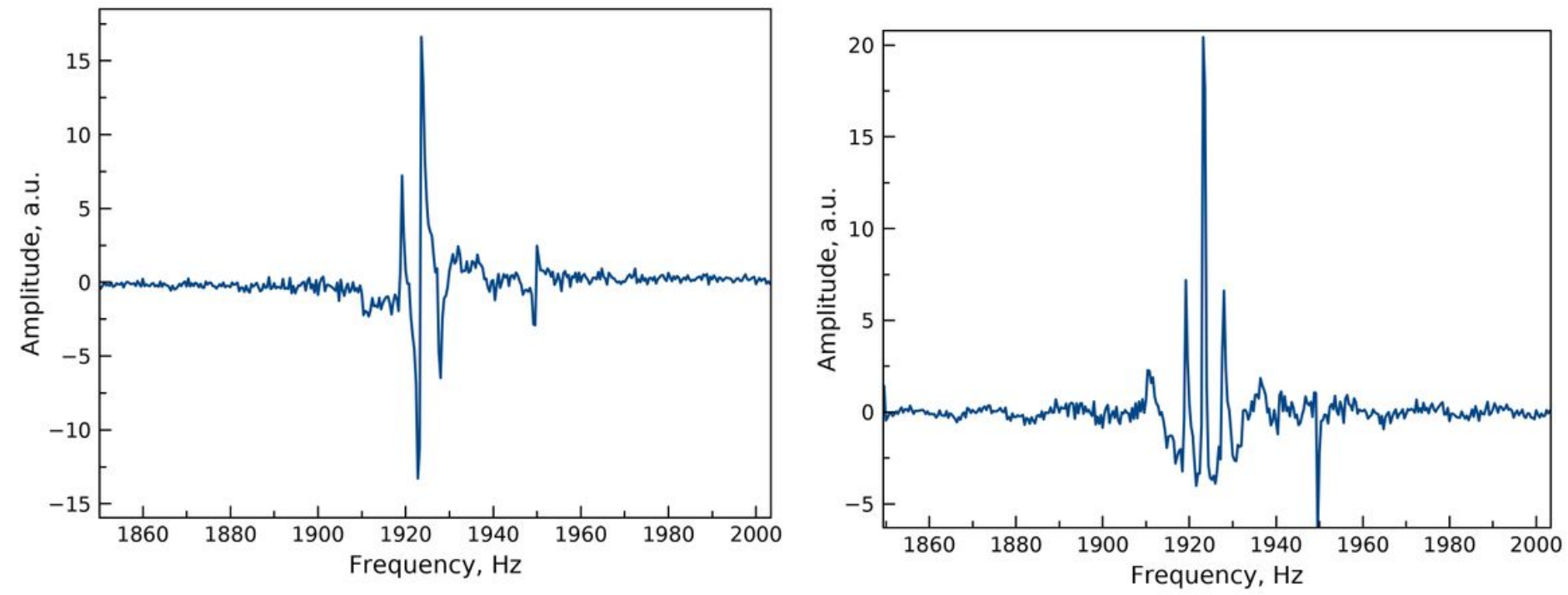

Figure 3

The real component of the NMR-spectrum of 2,2,2-trifluoroethanol in the Earth field without (left) and with (right) "dead time" correction (single scan). The central line corresponds to the $\mathrm{OH}$-group of alcohol and a small amount of water. In the region of $1950 \mathrm{~Hz}$ one can see interference from higher harmonics of industrial $50 \mathrm{~Hz}$.

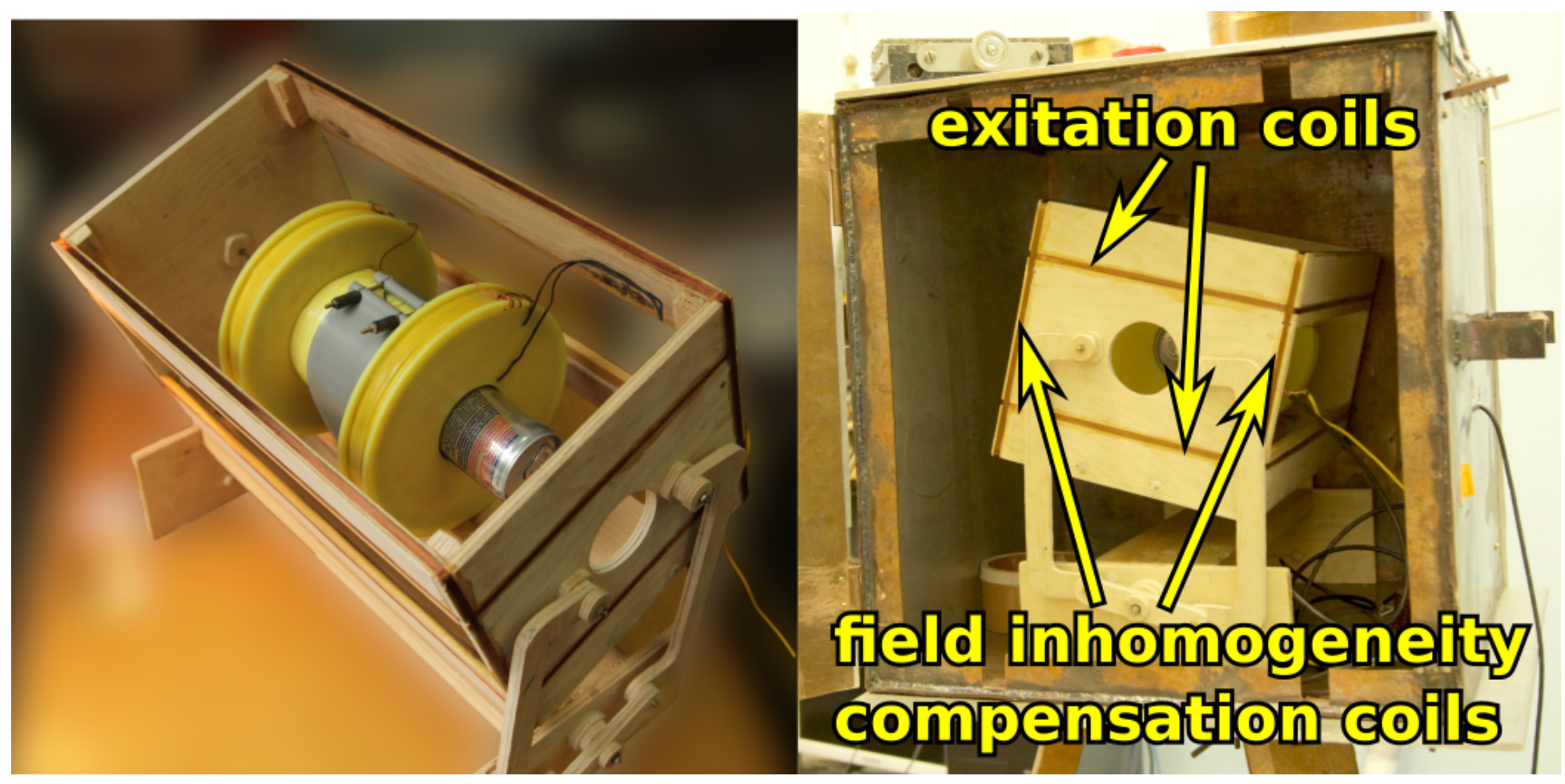

Figure 4

The NMR sensor in its frame (left); the sensor and indicated additional coils in the shielding cube (right). 

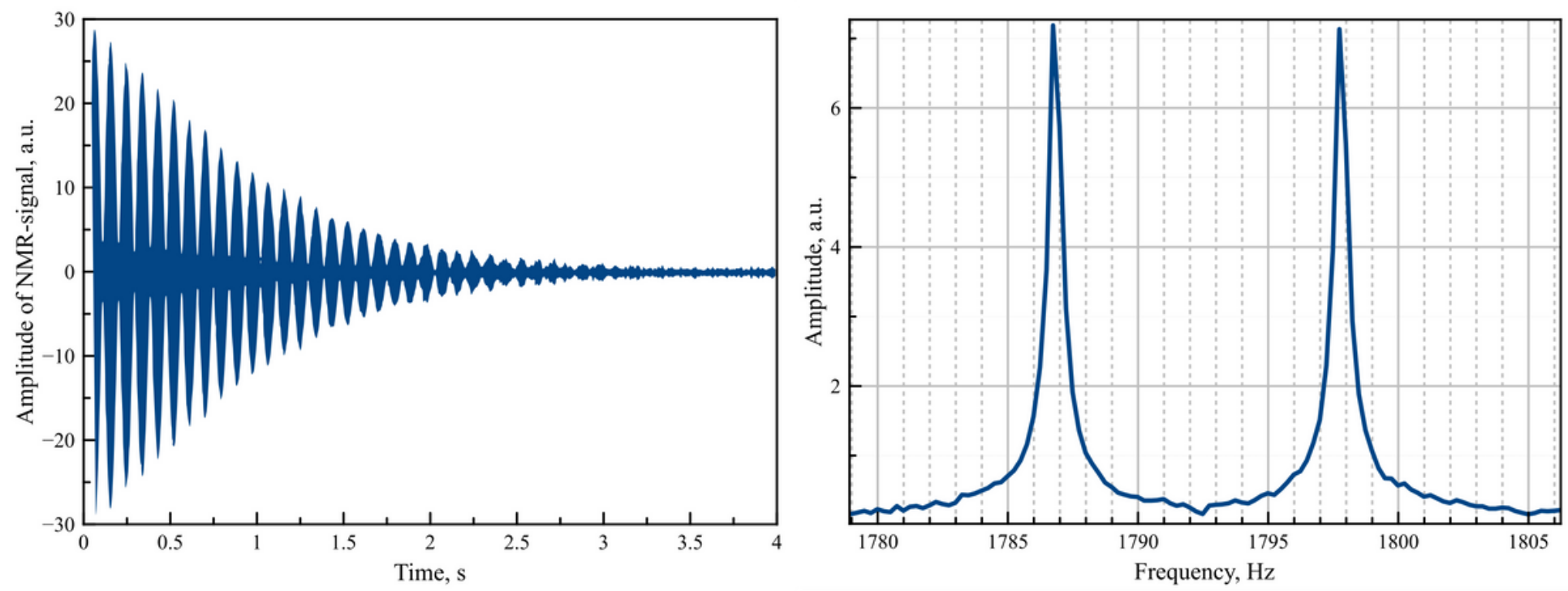

Figure 5

The trimethyl phosphate signal (single scan) in Earth magnetic field (a) and its spectrum (b).

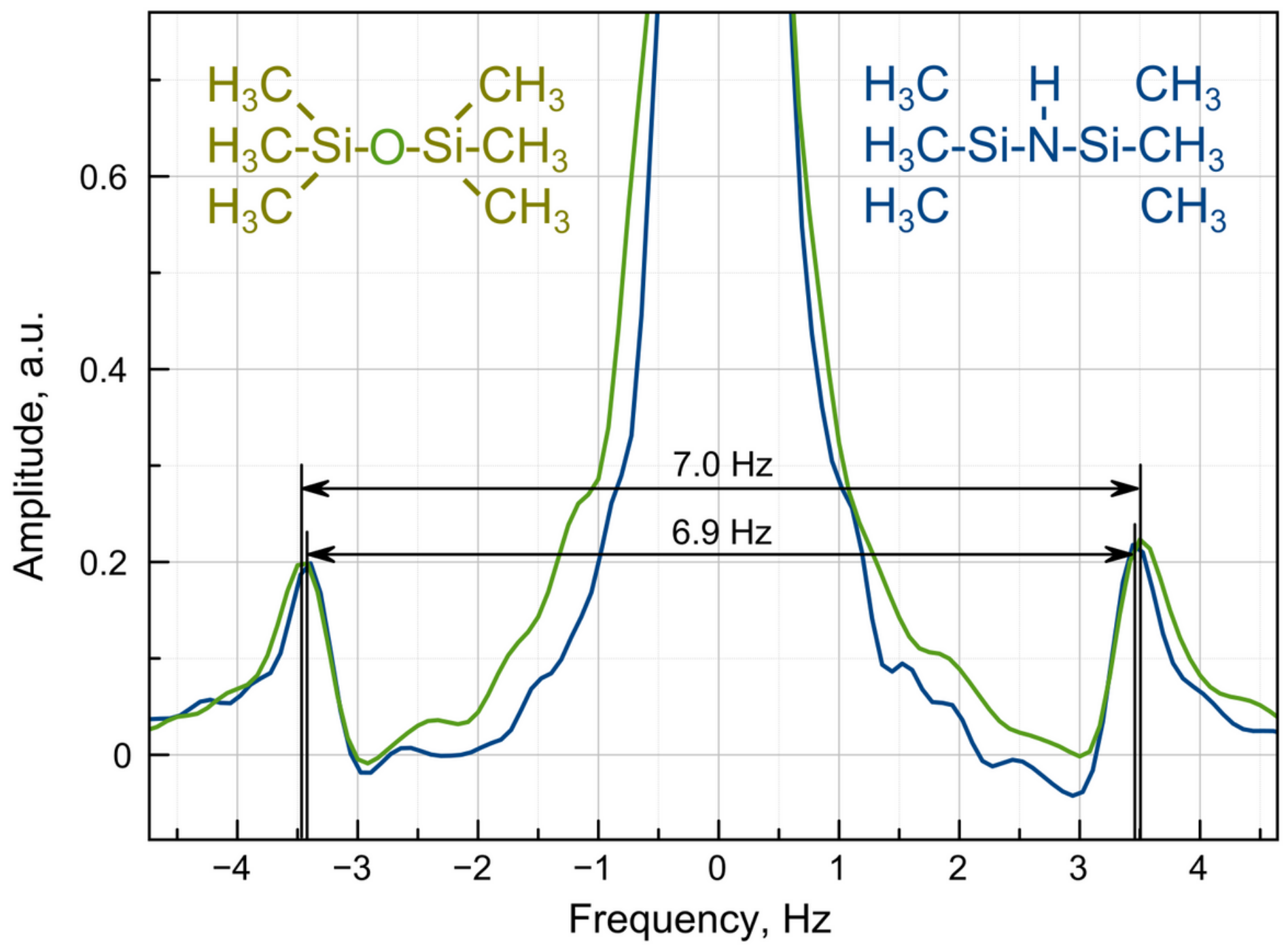


Figure 6

Proton spectra of hexamethyldisilazane (blue) and hexamethyldisiloxane (green) in the Earth magnetic field, 20 scans. The central line is cut off, its amplitude is 10 a.u.

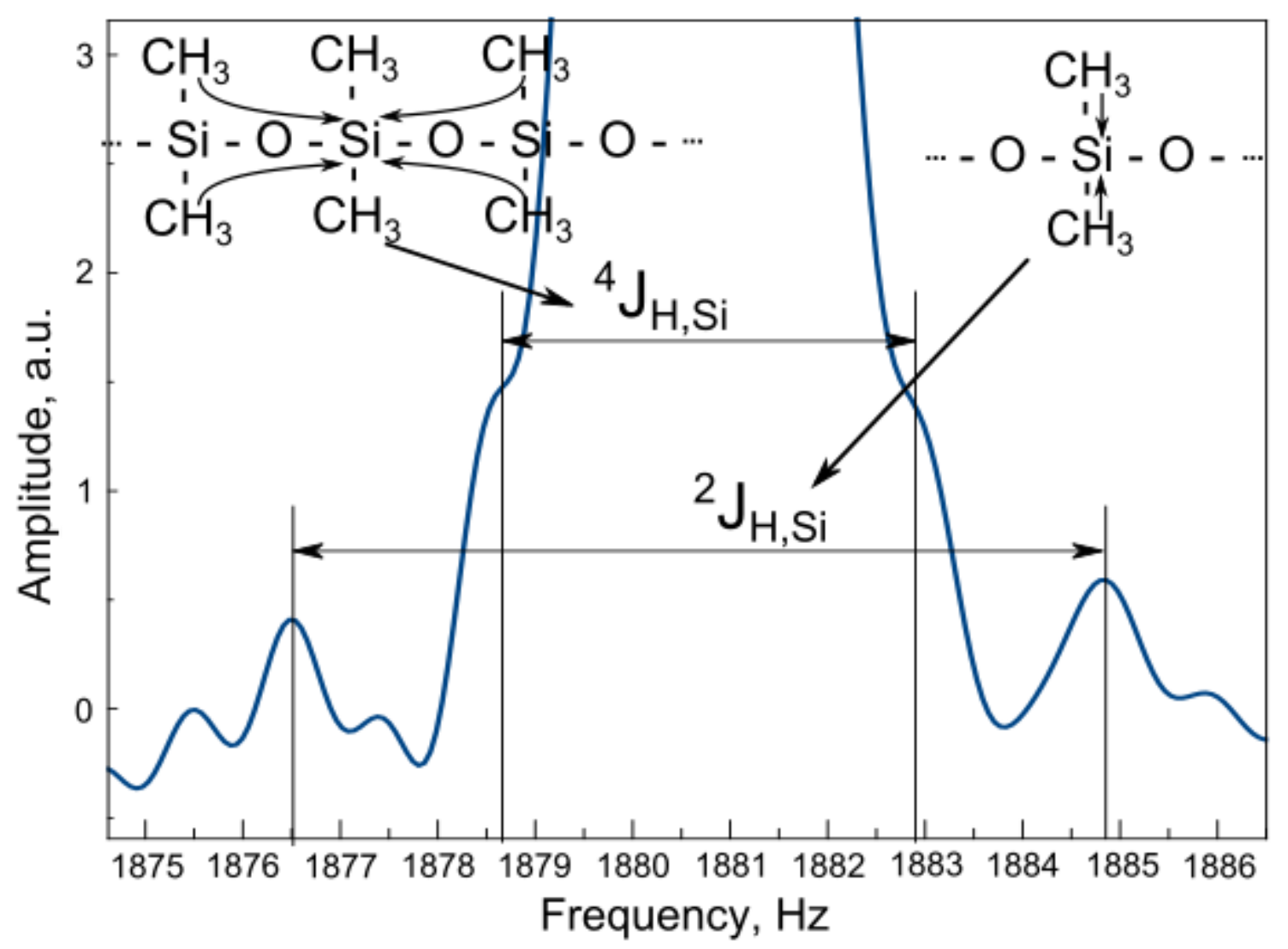

Figure 7

The proton spectra of polymethylsiloxane in the Earth magnetic field at $60^{\circ} \mathrm{C}$, eight scans. The central line is cut off, its amplitude is 10 a.u.
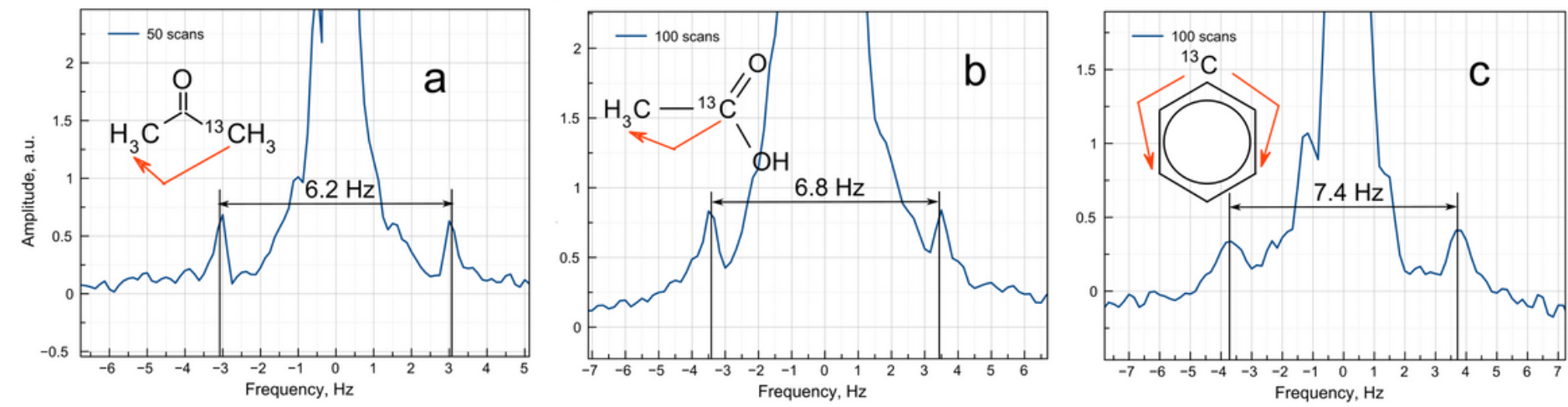

Figure 8 
NMR spectra of acetone (a), acetic acid (b) and benzene (c) in the Earth field. The figures show the Jcoupling constants. The amplitude of the main line is 100 a.u. 